\title{
Sexismo, temor a la evaluación negativa y comunicación destructiva en la pareja
}

\author{
Christian Enrique Cruz Torres ${ }^{1}$, Fredi Everardo Correa Romero ${ }^{2}$ y Joaquín \\ Alberto Padilla Bautista ${ }^{3}$ \\ Universidad de Guanajuato ${ }^{1,2}$ y Universidad Nacional Autónoma de \\ México ${ }^{3}$
}

El sexismo ambivalente propone que la hegemonía masculina se sostiene mediante la devoción hacia las madres, esposas y cuidadoras y mediante la hostilidad hacia mujeres que no cumplen dichos roles. Estos patrones incrementarían la comunicación violenta hacia las mujeres, efecto que se incrementaría por diferencias individuales como el temor a la evaluación social negativa (TESN). Se buscó identificar el impacto del TESN y los seis componentes del sexismo ambivalente sobre estilos de comunicación destructivos en la pareja, analizándose datos de 590 participantes jóvenes. Regresiones y modelos de ecuaciones estructurales confirman efectos del sexismo hostil y el TESN sobre la comunicación destructiva, pero solo para los hombres. Se discuten los efectos de cada componente del sexismo ambivalente sobre la comunicación destructiva.

Palabras clave: Sexismo, violencia en la pareja, violencia verbal, dependencia en la pareja, control sobre la pareja.

Sexism, fear of negative evaluation and destructive communication in the couple Ambivalent sexism proposes that male hegemony is sustained through devotion to mothers, wives and carers and through hostility towards women who do not fulfill these roles. These patterns would increase violent communication to women, an effect that would be increased by individual differences such as fear of negative social assessment (TESN). We sought to identify the impact of TESN and the six components of ambivalent sexism on destructive communication styles in the couple, analyzing data from 590 young participants. Regressions and models of structural equations confirm effects of hostile sexism and TESN on

1 Doctor en Psicología. Profesor investigador en la Universidad de Guanajuato. Dirección postal: Blvd. Puente milenio 1001, Fracción del Predio de San Carlos, C. P. 37670 . León, Guanajuato. México. Contacto: cassiel.79@gmail.com. https://orcid.org/0000-0002-4286-4697

2 Doctor en Psicología. Profesor investigador en la Universidad de Guanajuato. Dirección postal: Blvd. Puente milenio 1001, Fracción del Predio de San Carlos, C. P. 37670 . León, Guanajuato. México. Contacto: correafr@gmail.com. https://orcid.org/0000-0002-5856-7232

3 Doctor en Psicología. Investigador en la Coordinación General de Formación e Innovación educativa del Instituto Politécnico Nacional. Dirección postal: Universidad Nacional Autónoma de México. Facultad de Psicología, Av. Universidad 3004, Cd. Universitaria, Mezzanine del edificio “D” cubículo 8, C.P. 04510, Ciudad de México, México. Contacto: nembrod@ hotmail.com. https://orcid.org/0000-0002-2159-7286 
destructive communication, but only for men. The effects of each component of ambivalent sexism on destructive communication are discussed.

Keywords: Sexism, couple violence, verbal violence, couple dependency, control over the couple.

\section{Sexismo, medo de avaliaçáo negativa e comunicaçáo destrutiva no casal}

O sexismo ambivalente propóe que a hegemonia masculina seja sustentada pela devoção às mães, esposas e cuidadores e pela hostilidade para as mulheres que náo cumprem esses papéis. Esses padrôes aumentariam a comunicação violenta com as mulheres, um efeito que seria aumentado pelas diferenças individuais, como o medo da avaliaçáo social negativa (TESN). Buscamos identificar o impacto do TESN e os seis componentes do sexismo ambivalente em estilos de comunicação destrutiva no casal, analisando dados de 590 participantes jovens. Regressôes e modelos de equaçóes estruturais confirmam os efeitos do sexismo hostil e TESN na comunicação destrutiva, mas apenas para os homens. Os efeitos de cada componente do sexismo ambivalente na comunicação destrutiva são discutidos.

Palavras-chave: Sexismo, violência conjugal, violência verbal, dependência de casal, controle sobre o casal

Sexisme, peur de l'évaluation négative et communication destructive dans le couple Le sexisme ambivalent propose que l'hégémonie masculine soit soutenue par le dévouement envers les mères, les épouses et les soignants et par l'hostilité envers les femmes qui ne remplissent pas ces rôles. Ces modèles augmenteraient la communication violente aux femmes, un effet qui serait accru par des différences individuelles telles que la peur de l'évaluation sociale négative (TESN). Nous avons cherché à identifier l'impact du TESN et des six composantes du sexisme ambivalent sur les styles de communication destructeurs dans le couple, en analysant les données de 590 jeunes participants. Les régressions et les modèles d'équations structurelles confirment les effets du sexisme hostile et du TESN sur la communication destructive, mais seulement pour les hommes. Les effets de chaque composante du sexisme ambivalent sur la communication destructive sont discutés.

Mots clés: sexisme, violence conjugale, violence verbale, dépendance de couple, contrôle sur le couple 
Una forma común de analizar las relaciones estructurales entre mujeres y hombres es el microsistema de la pareja heterosexual, principal espacio en el que se ejerce violencia contra las mujeres. Estudios en México y alrededor del mundo muestran que la violencia en las relaciones de pareja presenta incidencias altas que no se ven disminuidas a pesar de los esfuerzos por combatirla. De acuerdo con la organización mundial de la salud, entre el $25.8 \%$ y el $33.9 \%$ de las mujeres de América Latina reportan haber sufrido alguna forma de violencia física por parte de alguna de sus parejas a lo largo de su vida, presentando importantes repercusiones sobre su salud física y mental (WHO, 2013). En 2003 se estimaba que el $41 \%$ de las mujeres en México habían sufrido alguna forma de violencia en su relación de pareja más reciente, siendo la violencia psicológica (19.6\%) y la física (9.8\%) las más comunes (INSP, 2003). En 2006, con la misma encuesta, se observó que el porcentaje de mujeres que sufrieron alguna forma de violencia en los 12 meses previos al levantamiento de estos índices se incrementó al 42.9\%, siendo nuevamente la violencia psicológica $(28.5 \%)$ y la física $(16.5 \%)$ las de mayor prevalencia. En estudios más recientes (INEGI, 2013), el $44.9 \%$ de las mujeres en México reporta haber sufrido alguna forma de violencia a lo largo de su actual relación de pareja. De estas mujeres, el $89.2 \%$ reportan haber sufrido violencia emocional (e.g. insultos, amenazas, humillaciones) y el $11.7 \%$ agresiones físicas. Buena parte de los estudios se enfocan en parejas que cohabitan, pero también se presenta en las relaciones de noviazgo, donde el 15\% de las y los jóvenes que conformaron la muestra del Instituto Mexicano de la Juventud (2008) había sufrido algún incidente de violencia física en su relación de pareja vigente. Dentro de quienes reportan haber vivido violencia, la proporción de mujeres es mayor (61.4\%). Con respecto a la violencia psicológica, el $76 \%$ reporta haber sufrido algún incidente de este tipo de violencia. 
Buena parte de la atención de la sociedad se ha centrado en la violencia física, pero como puede verse, la forma más común de violencia es la psicológica. Sufrir violencia psicológica, en particular violencia verbal, se ha asociado con un mayor riesgo de padecer trastornos crónicos, una mala salud en general y síntomas de depresión en hombres y mujeres (Coker, et. al, 2002; García-Moreno, 2000). La violencia psicológica genera por sí misma consecuencias que ameritan prestarle atención, pero debe considerarse además que es un antecedente común de otras formas de violencia (Follingstad, et. al, 1990), las cuales a su vez generan necesariamente aún más violencia psicológica (Torres, 2001).

La violencia psicológica puede presentarse en diferentes formas, pero el principal medio para su ejercicio es la comunicación verbal (Alonso et al., 2010). Este es un proceso difícil de abarcar, pero para estudiarlo, Sánchez-Aragón y Díaz-Loving (2003) han propuesto un modelo de diferentes estilos que se asumen como tendencias estables de comunicación en la pareja. Mediante un listado de descriptivos en el cual las personas evaluaban las formas en que se comunicaban regularmente con su pareja, fue posible identificar con análisis factoriales diferentes estilos de comunicación en parejas mexicanas, entre los cuales se identifican tres que coinciden con la idea de la comunicación verbal como un medio para el ejercicio de la violencia psicológica. El estilo violento instrumental refiere a un estilo de comunicación amenazante, agresiva, atacante y conflictiva; el estilo hiriente se caracteriza por los descriptivos injusto, humillante, grosero y ofensivo; y el estilo evitante se identifica por los descriptivos seco, frío, severo, intolerante y distante.

Uno de los factores ya identificado como antecedente de la violencia hacia las mujeres en relaciones de pareja es el sexismo ambivalente, un esquema de estereotipos que asumen roles tanto de superioridad de los hombres como de complementariedad entre hombres y mujeres (Glick y Fiske, 1996). Swin y Hayers (2009) definen el sexismo como actitudes, creencias y comportamientos individuales, institucionales y culturales que reflejan una evaluación negativa hacia ciertos individuos basados en su identificación sexo-genérica, en la cual se apoya la creencia de un 
estatus diferencial entre hombres y mujeres. Para Expósito et al. (1998), el sexismo es toda evaluación (en las dimensiones afectiva, cognitiva y conductual) que se haga de una persona atendiendo a la categoría sexual fenotípica a la que pertenece, tanto si es negativa como positiva, y tanto si se refiere al hombre como a la mujer.

Glick y Fiske (2001) plantean dos componentes del sexismo: hostil y benevolente. El sexismo hostil suele dirigirse hacia aquellas mujeres que se piensa buscan tener el poder de los hombres ya sea de manera abierta u oculta, a través del feminismo, de dedicarse a sus carreras profesionales y no cumplir con roles tradicionales, o bien, aquellas que se perciben como seductoras. Alude al desagrado o antipatía hacia mujeres que se perciben como "usurpadoras del poder de los hombres" (Glick y Fiske, 2001, p.109).

Por otro lado, el sexismo benevolente se presenta hacia mujeres que refuerzan los estereotipos tradicionales de género y cuya identidad podría centrarse en sus relaciones con los hombres como esposas, madres u objetos románticos antes que mujeres. Subyace la idea de que las mujeres que cumplen con roles tradicionales merecen ser depositarias de protección y afecto. Se les percibe como seres que deben ser resguardados, amados, y cuyo amor es el complemento de amor del hombre. Ambos componentes del sexismo funcionan como un programa de reforzamiento (sexismo benevolente) y castigo (sexismo hostil) para mantener el estatus quo. Si cumplen con los roles tradicionales son tratadas como débiles pero merecedoras de cuidado (sexismo benevolente), pero si se les percibe como fuertes, entonces no merecen buen trato (Glick y Fiske, 2001).

Estos componentes del sexismo se dividen en subtipos. El sexismo hostil se divide en paternalismo dominante, diferenciación competitiva de género y hostilidad heterosexual; mientras que el sexismo benevolente está conformado por el paternalismo protector, la diferenciación complementaria de género y la intimidad heterosexual

En el sexismo hostil, el paternalismo dominante, (Glick y Fiske, 1996) hace una analogía entre el trato del padre hacia las/los hijos y las acciones que, desde una mirada sexista, los hombres deben tener hacia 
las mujeres. El segundo componente, diferenciación competitiva de género, es una forma de justificar el poder estructural de los hombres, partiendo de la creencia de que solo ellos poseen las habilidades necesarias para gobernar los organismos sociales importantes, legitimando la jerarquía inferior de las mujeres (Glick y Fiske, 1996). Estas creencias le permiten a un hombre en particular aumentar su autoestima al asociarla con la identidad de los hombres en general. Finalmente, la hostilidad heterosexual (Glick y Fiske, 1996) hace que los hombres se miren como vulnerables ante las mujeres y estas sean evaluadas como tentadoras y tratadas de manera hostil (Glick y Fiske, 1996).

En el sexismo benevolente se encuentra el paternalismo protector, que alude que las mujeres requieren de un hombre proveedor y protector que satisfaga sus necesidades, puesto que a ellas se les percibe como frágiles y dependientes. Este componente del sexismo ayuda a los hombres a mantener su autoimagen positiva de proveedores.

El segundo componente del sexismo benevolente, la diferenciación complementaria de género, involucra la creencia de que las mujeres poseen características como la pasividad y la capacidad de acompańamiento, mientras que los hombres poseen otras como impulsividad y agencia, encasillando a mujeres y hombres en roles estereotipados. El tercer componente, la intimidad heterosexual, alude a que las relaciones heterosexuales monógamas son las únicas donde se puede encontrar intimidad, cercanía y afecto; implicando la dependencia de los hombres hacia las mujeres como compañeras indispensables.

Las creencias de que las diferencias entre hombres y mujeres son extremas, más grandes entre grupos que dentro de los grupos; que los hombres son superiores a las mujeres en los aspectos más relevantes para justificar un acceso prioritario a los medios de poder; así como la idea de que las mujeres usan su atractivo sexual para ganar poder sobre ellos, son el principal sustento del sexismo hostil (Glick y Fiske, 2001). Glick et al. (1997) muestran que los hombres que presentan mayores puntajes en sexismo ambivalente suelen tener visiones más estereotipadas de las mujeres, tendiendo a categorizarlas como buenas o malas. Además, hombres con puntajes más altos de sexismo hostil predicen 
actitudes negativas hacia las mujeres que se enfocan en sus carreras profesionales; mientras que un puntaje alto de sexismo benevolente predice actitudes positivas hacia las mujeres que realizan trabajo no remunerado en sus hogares. Respecto a las mujeres, se observa que es más probable que en los países más sexistas aprueben el sexismo benevolente de su pareja y de otros, esto cuando dependen económicamente de sus parejas (Glick y Fiske, 2001), además de ser menos probable que protesten contra el poder de los hombres.

Varios estudios previos han mostrado que el sexismo ambivalente funciona como justificador de la violencia contra las mujeres (Glick et al., 2002) incluso en poblaciones de jóvenes y en mujeres, en quienes esperaríamos menos efectos derivados de estereotipos tradicionales de género (Garaigordobil et al., 2013). Menos estudios se han enfocado en los efectos directos de los componentes del sexismo ambivalente sobre el ejercicio de la violencia en la pareja. Por ejemplo, los resultados de Overall et al. (2011), muestran que el sexismo ambivalente puede afectar la comunicación verbal, reduciendo su efectividad para llegar a acuerdos e incrementando su hostilidad; en específico, sus resultados muestran que estos efectos se presentan en parejas donde el hombre ostenta niveles más altos de sexismo hostil. Sin embargo, todos estos estudios presentan, a nuestro parecer, una limitación con respecto a la correspondencia entre el modelo teórico y la medición del sexismo ambivalente. Glick y Fiske (1996) proponen una estructura teórica de seis componentes que se agrupan a su vez en las dimensiones de sexismo hostil y benevolente, pero su instrumento, aunque confiable y ampliamente utilizado en el mundo, distingue únicamente entre las dimensiones globales de sexismo hostil y benevolente, sin identificar los tres componentes internos de cada dimensión. Aunque esta distinción ya fue un aporte fundamental para la mejor comprensión de los estereotipos de género, resulta necesario saber si el análisis de cada componente al interior del sexismo hostil y benevolente ofrece nueva información, en este caso, respecto de los efectos del sexismo sobre la violencia en las relaciones de pareja heterosexuales. Previamente, el instrumento de Cruz et al. (2005) en una muestra en México, había 
identificado las seis subdimensiones del sexismo ambivalente obteniendo adecuados niveles de consistencia interna, confirmando además que estas se agrupan en dos dimensiones de segundo orden congruentes con el sexismo hostil y el benevolente. Niveles similares de consistencia interna y la misma capacidad de diferenciar las subdimensiones del sexismo hostil y benevolente han sido encontradas también al analizar muestras de habitantes en Perú (Rottenbacher, 2012).

Un factor que podría incrementar los efectos previamente identificados del sexismo sobre el ejercicio de la violencia es el temor a la evaluación social negativa (TESN), definido por Watson y Friend (1969) como un temor hacia la valoración de los demás y a las expectativas que se generan alrededor de la misma, donde la desaprobación causa un malestar exagerado en el individuo al grado que puede evitar los estímulos que le parecen amenazantes y abandonar sus actividades cotidianas. En países más sexistas las mujeres aprueban más el sexismo (Glick y Fiske, 2001), lo cual es relevante en un contexto como México, con un Índice de Desigualdad de Género (IDG) 0.393 en 2012 (Programa de Naciones Unidad para el Desarrollo, 2017). Sin embargo, es factible también que las personas puedan estar motivadas para evitar mostrar sus prejuicios sexistas, identificándose que existen motivaciones internas (e.g. cuando esos prejuicios no se expresan por ser opuestos al autoconcepto del individuo) y externas (e.g. cuando no se expresan para evitar una posible sanción social) (Klonis et al., 2005).

Este mismo fenómeno puede identificarse también en el prejuicio hacia las personas afroamericanas (Plant y Devine, 1998). En ambos estudios (Klonis et al., 2005; Plant y Devine, 1998), los reactivos de motivación externa para evitar mostrar sus prejuicios implican que mostrarlos es socialmente sancionable, y es en ese contexto que las personas con altos niveles de TESN se reportan más motivadas, pero solo externamente, para evitar mostrar sus prejuicios. Sin embargo, en un contexto donde los estereotipos sexistas son la norma, los individuos con altos niveles de TESN podrían mostrar un mayor respaldo a estos estereotipos para evitar ser sancionados socialmente. Contrastando experimentalmente escenarios públicos y privados, el estudio 
de Lambert y colaboradores (2003), encuentra que personas con altos niveles de TESN tienden a hacer un mayor uso de información estereotípica para evaluar a otras personas, especialmente cuando estas evaluaciones se dan en escenarios públicos.

Respecto al vínculo entre la violencia en la pareja y el TESN, el estudio de Hanby et al. (2012), muestra que hombres con altos niveles de TESN tienden a reportar también niveles más altos de agresión física y psicológica hacia sus parejas. Además, el estudio de Darcy et al. (2005) muestra que las personas con niveles altos de ansiedad social tienden a reportar niveles más altos de dependencia hacia sus parejas. Aunado a lo anterior, se observa que estereotipar a las mujeres como un grupo de menor estatus que el de los hombres, y al mismo tiempo considerarlas como únicas depositarias del afecto masculino, suele fomentar y normalizar la codependencia y la violencia contra ellas (Fiske et al., 1999).

El presente estudio tiene por objetivo identificar el impacto del TESN y el sexismo ambivalente y sus seis componentes independientes sobre el ejercicio de estilos de comunicación destructivos en la pareja. Se plantea como hipótesis que sexismo y TESN se asociarán positivamente con el ejercicio de estilos de comunicación destructivos, aunque se analizan adicionalmente posibles efectos diferenciados de cada componente del sexismo ambivalente y diferencias de estos efectos para hombres y mujeres.

\section{Método}

\section{Participantes}

Participaron 164 hombres y 426 mujeres, estudiantes universitarios, residentes del área metropolitana de la Ciudad de México, con una edad promedio de 20.4 años $(D E=2.7)$. El 54\% declaró estar involucrado en una relación de pareja al momento de participar en el estudio y haber vivido entre 1 y 35 relaciones de pareja hasta el momento del estudio, con un promedio de 5.7 relaciones $(D E=4.7)$. 


\section{Instrumentos}

Sexismo Ambivalente. Se utilizó una versión revisada del Cuestionario de Medición del Sexismo Ambivalente (Cruz et al., 2005), compuesta de 25 reactivos. Como resultado de un análisis factorial de máxima verosimilitud, el instrumento muestra una estructura de seis factores congruentes con la teoría del sexismo ambivalente (Glick y Fiske, 1996), que conserva 19 de los 25 reactivos originales y explica el $50.06 \%$ de la varianza. Aunque el indicador de bondad de ajuste para este análisis muestra discrepancias de ajuste significativas $\left(\mathrm{i}^{2}=113.57\right.$; $g l=60 ; p<.001)$, un análisis factorial confirmatorio mediante ecuaciones estructurales confirma los mismos factores con indicadores de bondad de ajuste adecuados, indicando niveles altos de la varianza total explicada a través del modelo teórico $(G F I=.94)$, diferencias significativas del modelo propuesto en comparación con un modelo de relaciones nulas entre sus elementos $(C F I=.93)$, una proporción aceptable de residuos de varianza una vez controlada la varianza de las relaciones entre los elementos del modelo $(R M R=.05)$, y un adecuado nivel de parsimonia en el modelo ( $R M S E A=.044$, intervalo de confianza al $90 \%$ mín=.036/max=.052). Sin embargo, se observan valores significativos para el indicador $J i^{2}\left(J i^{2}=136.36 ; g l=72 ; p<.001\right)$, que indicarían discrepancias importantes entre las relaciones planteadas entre los elementos del modelo teórico y las observadas en los datos analizados. Considerando que el resto de los indicadores se encuentran dentro de límites aceptables (Levy y Varela, 2003) y que algunos de ellos como el GFI constituyen correcciones del valor $J i^{2}$ basadas en el tamańo de la muestra (Bentler y Bonett, 1980; Kline, 2011), es probable que los valores de $\mathrm{Ji}^{2}$ se encuentren artificialmente incrementados por el tamaño de la muestra. La consistencia interna del factor diferenciación competitiva de género fue $\alpha=.74$; para hostilidad heterosexual $\alpha=.80$; para paternalismo dominante, $\alpha=.68$; para paternalismo protector, $\alpha=.80$; para intimidad heterosexual, $\alpha=.71$; y para diferenciación complementaria de género $\alpha=.62$.

TESN. Se utilizó la traducción al español de Cruz et. al. (2013) del instrumento de Leary (1983), mostrando una consistencia interna 
de $\alpha=.92$, con ocho reactivos en formato tipo Likert con seis opciones de respuesta que se agrupan en un solo factor (e.g. A menudo me da miedo que la gente note mis defectos).

Estilos de comunicación destructiva. Se evaluaron mediante una selección de los reactivos del instrumento de Estilos de Comunicación de Sánchez Aragón y Díaz Loving (2003) que presentaban cargas factoriales más altas. Mediante un análisis factorial exploratorio de máxima verosimilitud se obtuvieron cinco factores, dos de los cuales denotan estilos de comunicación destructiva y fueron los únicos analizados en el presente proyecto. El primero fue denominado comunicación violenta $(\alpha=.90)$, compuesto por 16 reactivos que hablan tanto de la posibilidad de hacer daño (i.e. atacante, conflictivo, agresivo/a, humillante, hiriente, amenazante, insolente, destructivo/a, grosero/a, y rechazante), como de la intención de controlar a la pareja (i.e. dictatorial, controlador/a, inconforme, limitante, inquisitivo/a, y criticón/a). El segundo factor fue denominado pasivo-agresivo $(\alpha=.79)$, compuesto de tres reactivos que hablan de rechazo y la negación del afecto (i.e. frío/a, seco/a, distante).

Todos los instrumentos se presentaron en formato tipo Likert con opciones de respuesta de 1 (Totalmente en desacuerdo) a 6 (Totalmente de acuerdo). Finalmente, se preguntaban datos generales (edad, sexo, pareja, etc.).

Una vez confirmadas las estructuras de cada factor y verificada su consistencia interna, los reactivos se promediaban en un nuevo indicador para cada factor.

\section{Procedimiento}

El muestreo fue no probabilístico, por conveniencia. Se invitaba a las y los participantes a resolver el cuadernillo voluntariamente, informando que sus datos serían confidenciales, que no se solicitaban datos que permitieran su identificación personal, y que no habría ni sanciones por no participar no recompensas por participar. Previa autorización de los participantes, la aplicación se realizaba en salones de clase, durante aproximadamente 20 minutos. 


\section{Resultados}

Los puntajes en el estilo de comunicación violento fueron de $M=1.85$ ( $\mathrm{DE}=.99)$, sin diferencias estadísticamente significativas entre hombres y mujeres $(t=-.358, g l=588, p=.72)$. Para el estilo pasivo agresivo el puntaje fue $M=1.78(D E=.71)$, sin diferencias estadísticamente significativas entre hombres y mujeres ( $t=.254, g l=588, p=.799)$. Se realizaron comparaciones de promedios entre hombres y mujeres para cada componente del sexismo ambivalente y el TESN, encontrándose en todos los casos promedios por debajo de la media teórica y diferencias estadísticamente significativas, como puede verse en la Tabla 1.

\section{Tabla 1}

Comparación de promedios entre hombres y mujeres para los factores de sexismo ambivalente y el TESN

\begin{tabular}{lcccccc}
\hline Factores & Sexo & $M$ & $D E$ & $t$ & $g l$ & $p$ \\
\hline Diferenciación & Hombres & 1.58 & .87 & 4.14 & 212.83 & $<.001$ \\
competitiva de género & Mujeres & 1.27 & .54 & & & \\
& Hombres & 2.95 & 1.28 & & & \\
Hostilidad heterosexual & Mujeres & 2.32 & 1.08 & 5.59 & 257.89 & $<.001$ \\
& Hombres & 2.80 & 1.26 & & & \\
Paternalismo protector & Mujeres & 2.26 & 1.19 & & & \\
& Hombres & 2.01 & 1.16 & 388 & $<.001$ \\
Intimidad heterosexual & Mujeres & 1.61 & .86 & & & \\
\multirow{2}{*}{$\begin{array}{l}\text { Diferenciación } \\
\text { complementaria de género }\end{array}$} & Mujeres & 3.40 & 1.18 & -2.16 & 588 & .031 \\
& Hombres & 2.16 & 1.02 & & & \\
Paternalismo dominante & Mujeres & 1.63 & .83 & 5.85 & 250.57 & $<.001$ \\
& Hombres & 2.59 & 1.21 & & & \\
TESN & Mujeres & 2.91 & 1.29 & -2.78 & 588 & .005 \\
\hline
\end{tabular}

Nota: En los casos donde la prueba de equivalencia de varianzas de Levene señalaba varianzas no equivalentes, las comparaciones se realizaron con los ajustes de Welch (Welch, 1947; Zimmerman, 2004) a la prueba $t$ de Student. 
Para probar los efectos del TESN y los factores del sexismo ambivalente sobre los estilos de comunicación violento y controlador se utilizaron regresiones lineales por pasos, descartando los factores que presentaran valores de $F$ con $p>.05$.

Se observan porcentajes de varianza explicada de apenas el $3 \%$ para el estilo pasivo-agresivo $\left(r^{2}=.038 ; F=11.56, g l=2,587, p<.001\right)$ y del $13 \%$ para el estilo violento $\left(r^{2}=.134 ; F=22.57, g l=4,585, p<.001\right)$. Para el estilo pasivo-agresivo la explicación está dada por efectos positivos del TESN $(\beta=.145 ; t=3.54, \mathrm{p}<.001)$ y la intimidad heterosexual $(\beta=.114 ; t=2.78, p=.006)$. Para el estilo violento se observaron efectos positivos del TESN $(\beta=.276 ; t=7.06, p<.001)$, la hostilidad heterosexual $(\beta=.179 ; t=4.29, p<.001)$, y la intimidad heterosexual $(\beta=.116$; $t=2.65, p=.008)$, más efectos negativos del factor diferenciación complementaria de género $(\beta=-.124 ; t=-2.94, p=.003)$.

Se analizó posteriormente la posibilidad de que estos modelos tuvieran comportamientos diferentes en hombres y mujeres, encontrando diferencias notables. Para los hombres, la varianza explicada para el estilo pasivo-agresivo alcanza el $14 \%\left(r^{2}=.147 ; \quad F=13.88\right.$, $g l=2,161, p<.001)$, explicada por la hostilidad heterosexual $(\beta=.436$; $t=5.269, p<.001$ ), más un efecto negativo del paternalismo protector ( $\beta=-.199 ; t=-2.407, p=.017)$. Para las mujeres, la varianza explicada del estilo pasivo-agresivo alcanza apenas el 3\% $\left(r^{2}=.031 ; F=13.62\right.$, $g l=1,424, p<.001)$, derivado de efectos positivos del TESN $(\beta=.176$; $t=3.69, p<.001)$.

Para el estilo violento, en los hombres, el porcentaje de explicación se incrementa al $24 \%\left(r^{2}=.241 ; F=12.61, g l=4,159, p<.001\right)$, derivado de la hostilidad heterosexual $(\beta=.278 ; t=3.25, p=.011)$, seguido del TESN $(\beta=.277 ; t=3.93, p<.001)$, el paternalismo dominante $(\beta=.281$; $t=3.12, p=.002)$, y efectos negativos del paternalismo protector $(\beta=-$ .294; $t=-3.41, p=.001)$. Para las mujeres, la explicación del estilo violento alcanzó el 10\% ( $\left.r^{2}=.100 ; F=23.53, g l=2,423, p<.001\right)$, dado por el TESN $(\beta=.272 ; t=5.87, p<.001)$, y la hostilidad heterosexual $(\beta=.142 ; t=3.07, p=.002)$. 
Para todos los modelos, la tolerancia se mantuvo por encima de .59 y el factor de inflación de varianza por debajo de 1.7, denotando la ausencia de problemas de colinealidad.

Para poner a prueba un modelo más integral de los efectos del sexismo y el TESN sobre la comunicación violenta y pasivo-agresiva, se utilizó el modelado de ecuaciones estructurales mediante el software Amos versión 23 (Arbuckle, 2014), poniéndose a prueba efectos derivados del sexismo hostil o benevolente, como variables latentes, y de cada uno de sus componentes como variables observadas. Dadas las diferencias encontradas en los análisis de regresión, se realizaron estos análisis mediante la comparación multigrupos, analizando posibles diferencias tanto en la varianza explicada total de comunicación violenta y pasivo-agresivo, como en los efectos de TESN y los componentes de sexismo ambivalente.

La Figura 1 muestra un primer modelo para la comunicación pasivo-agresiva ejercida hacia la pareja, comparando a hombres y mujeres (para el modelo sin restricciones, $J i^{2}=82.97, g l=36, p<.001$; $R M R=.069 ; \quad G F I=.96 ; C F I=.95 ; \quad R M S E A=.047$, con un intervalo de confianza del 90\%, límite inferior .034, límite superior $=.060$ ). La comparación entre el modelo sin restricciones y el modelo de equivalencia métrica muestra diferencias estadísticamente significativas entre ambos modelos $\left(\Delta J i^{2}=14.43, g l=6, p=.025\right)$. Estas diferencias no se deben al modelo de medición del sexismo ambivalente, donde todos los efectos de las variables de sexismo presentan cargas significativas sobre las respectivas variables latentes de sexismo hostil y benevolente ( $C R$ mayores a 7.3, con valores de $p<.001$ ). Debe seńalarse el alto nivel de asociación entre ambas formas de sexismo tanto para los hombres $(C R=5.1, p<.001)$ como para las mujeres $(C R=7.66, p<.001)$, haciendo patente la función de ambos componentes como partes de un mismo sistema de creencias respecto a la inferioridad de las mujeres y la necesidad de tener control sobre ellas. Las diferencias entre hombres y mujeres se observan, en primer lugar, en la varianza de la comunicación pasivo-agresiva explicada por el modelo (16\% para los hombres, $3 \%$ para las mujeres). En el caso de los hombres, la comunicación 
pasivo-agresiva está explicada significativamente por un efecto negativo del sexismo benevolente $(C R=-1.98, p=.047)$, y un efecto positivo del sexismo hostil $(C R=2.67, p=.008)$, sin efectos significativos del TESN $(C R=1.51, p=.129)$. Para las mujeres, la explicación de la comunicación pasivo-agresiva está dada únicamente por el TESN $(C R=3.68, p<.001)$, sin efectos significativos para el sexismo hostil ni para el sexismo benevolente ( $C R$ menores a .6, con valores de $p>.59)$.

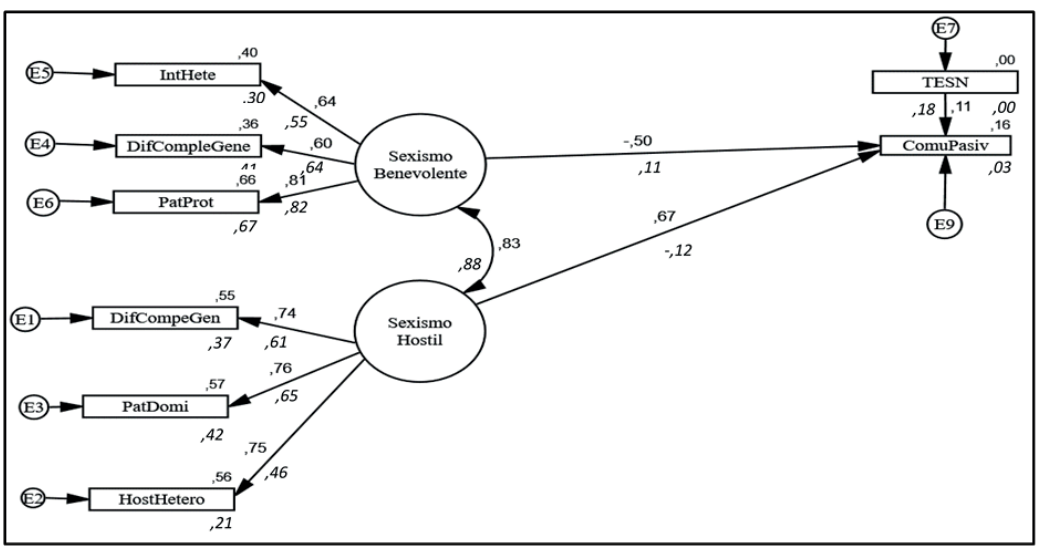

Figura 1. Modelo de ecuaciones estructurales de los efectos del sexismo ambivalente y el TESN sobre la comunicación pasivo-agresiva ejercida por hombres y mujeres. Los índices de los hombres se muestran por encima de cada trayectoria y variable, los índices de las mujeres se muestran en itálicas por debajo o a la izquierda de cada trayectoria y variable. Se muestran valores estandarizados.

Una revisión de las covarianzas restantes mostró efectos adicionales de la intimidad heterosexual sobre el TESN y la comunicación pasivo-agresiva, y de la hostilidad heterosexual sobre la comunicación pasivo-agresiva. Al incorporar esas trayectorias, presentes en la Figura 2, se observan mejores indicadores de bondad de ajuste $\left(\mathrm{J}^{2}=48.34, g l=30\right.$, $p=.018 ; R M R=.038 ; G F I=.98 ; C F I=.98 ; R M S E A=.03$, con un intervalo de confianza del $90 \%$, límite inferior .013 , límite superior $=.048$ ), 
manteniéndose las diferencias estadísticamente significativas entre los modelos de hombres y mujeres que indican que el mismo modelo no es equivalente para hombres y mujeres en términos de equivalencia métrica $\left(\Delta J i^{2}=16.08, g l=6, p=.013\right)$. Los niveles de varianza explicada de la comunicación pasivo-agresiva se incrementan al 20\% para los hombres y al $5 \%$ para las mujeres. Para las mujeres, estos niveles están dados únicamente por el TESN $(C R=3.5, p<.001)$ y la intimidad heterosexual ( $C R=2.06, p=.03$ ), con efectos no significativos para las variables latentes de sexismo hostil $(C R=.17, p=.86)$ y benevolente $(C R=-.43$, $p=.66)$. Para los hombres, la explicación se da por efectos positivos de la hostilidad heterosexual $(C R=3.41, p<.001)$ y la intimidad heterosexual $(C R=2.03, p=.04)$, que presenta efectos positivos sobre la comunicación pasivo-agresiva, en contraste con el efecto positivo del sexismo benevolente. Los efectos previamente identificados del sexismo hostil $(C R=.75, p=.45)$ y el sexismo benevolente $(C R=-1.94, p=.05)$ pierden significancia en este nuevo modelo.

Para los hombres, se observa además un efecto de la intimidad heterosexual sobre el TESN positivo y significativo, $(C R=2.26, p=.026)$, pero el efecto del TESN sobre la comunicación pasivo-agresiva no es estadísticamente significativo $(C R=1.14, p=.25)$. Para las mujeres se observa también un efecto de la intimidad heterosexual sobre el TESN $(C R=2.91, p=.004)$, con efectos del TESN significativos sobre la comunicación pasivo-agresiva $(C R=3.54, p<.001)$. 


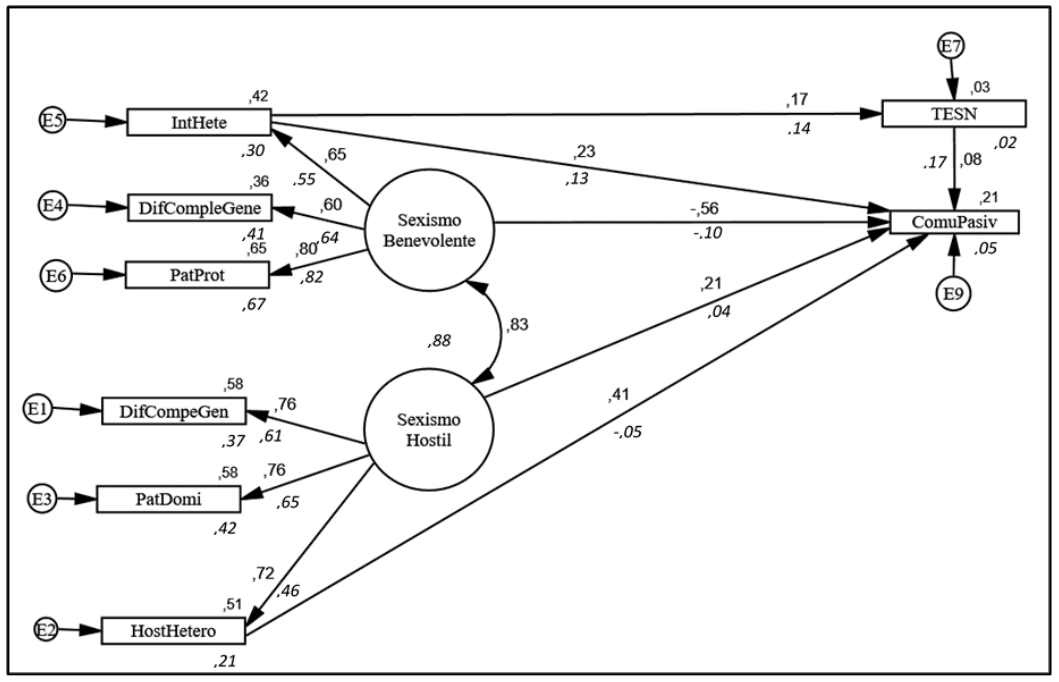

Figura 2. Modelo de ecuaciones estructurales de los efectos del sexismo ambivalente y el TESN sobre la comunicación pasivo-agresiva ejercida por hombres y mujeres, incorporando efectos directos de la intimidad heterosexual sobre el TESN y la comunicación pasivo-agresiva, y la hostilidad heterosexual sobre la comunicación pasivo-agresiva. Los índices de los hombres se muestran por encima de cada trayectoria y variable, los índices de las mujeres se muestran en itálicas por debajo o a la izquierda de cada trayectoria y variable. Se muestran valores estandarizados.

Se siguió la misma estrategia de análisis para la comunicación violenta. El primer modelo, presentado en la Figura 3, muestra adecuados indicadores de ajuste para el modelo sin restricciones $\left(J i^{2}=81.69\right.$, $g l=36, p<.001 ; R M R=.070 ; G F I=.96 ; C F I=.95 ; R M S E A=.046$, con un intervalo de confianza del $90 \%$, límite inferior .033, límite superior $=.060)$, con diferencias significativas para la equivalencia métrica entre hombres y mujeres $\left(\Delta J i^{2}=14.36, g l=6, p=.026\right)$. Para los hombres, la comunicación violenta alcanza una varianza explicada del $42 \%$, derivados de efectos positivos del sexismo hostil $(C R=3.73, p<.001)$, el TESN ( $C R=4.08, p<.001)$, y efectos negativos del sexismo benevolente 
$(C R=-2.89, p=.004)$. Para las mujeres, la explicación de la comunicación violenta es del 10\%, derivada únicamente del TESN ( $C R=6.09$, $p<.001)$.

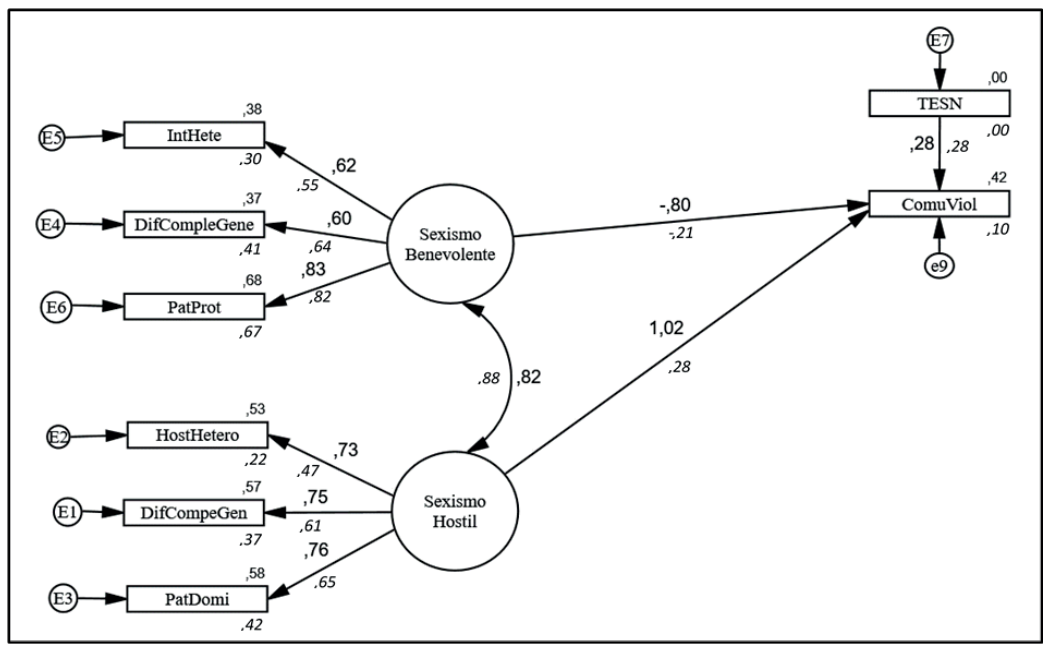

Figura 3. Modelo de ecuaciones estructurales de los efectos del sexismo ambivalente y el TESN sobre la comunicación violenta ejercida por hombres y mujeres. Los índices de los hombres se muestran por encima o a la derecha de cada trayectoria y variable, los índices de las mujeres se muestran en itálicas por debajo o a la izquierda de cada trayectoria y variable. Se muestran valores estandarizados.

La revisión de las covarianzas sobrantes sugiere nuevamente efectos directos de la intimidad heterosexual sobre el TESN y la comunicación violenta. Al incorporar estas trayectorias, presentadas en la Figura 4, se observan mejorías en la bondad de ajuste $\left(J i^{2}=54.05, g l=32, p=.009\right.$; $R M R=.040 ; G F I=.97 ; C F I=.97 ; R M S E A=.03$, con un intervalo de confianza del $90 \%$, límite inferior =.017, límite superior =.050), manteniéndose las diferencias de equivalencia métrica del modelo para las comparaciones entre hombres y mujeres $\left(\Delta J i^{2}=16.07, g l=6, p=.013\right)$. Para los hombres el nivel de varianza explicada de la comunicación 
violenta es del 58\%, manteniéndose significativos los efectos negativos del sexismo benevolente $(C R=-3.03, p<.001)$, el sexismo hostil $(C R=3.42, p<.001)$ y el TESN $(C R=3.95, p<.001)$, añadiéndose efectos positivos de la intimidad heterosexual sobre la comunicación violenta $(C R=2.79, \mathrm{p}=.005)$ y el TESN $(C R=2.22, p=.026)$. Para las mujeres, la varianza explicada de la comunicación violenta es del $12 \%$, derivada únicamente del TESN $(C R=5.80, p<.001)$ y la intimidad heterosexual $(C R=2.33, p=.019)$.

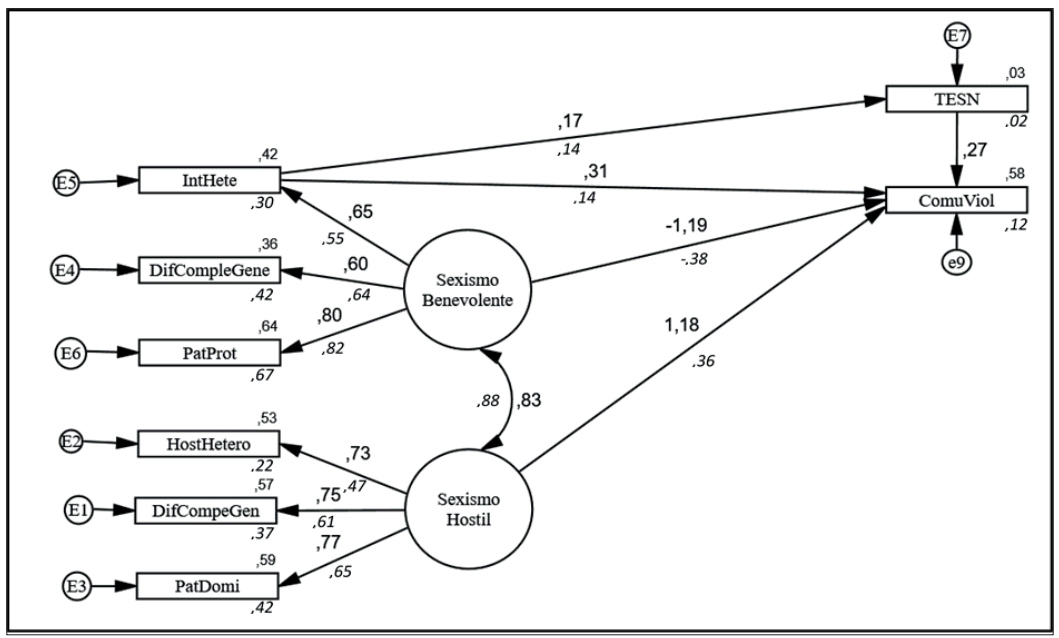

Figura 4. Modelo de ecuaciones estructurales de los efectos del sexismo ambivalente y el TESN sobre la comunicación violenta ejercida por hombres y mujeres, incorporando efectos directos de la intimidad heterosexual sobre el TESN y la comunicación violenta. Los índices de los hombres se muestran por encima de cada trayectoria y variable, los índices de las mujeres se muestran en itálicas por debajo o a la izquierda de cada trayectoria y variable. Se muestran valores estandarizados. 


\section{Conclusiones}

La medición de sexismo ambivalente utilizada en este estudio, (Cruz et al., 2005), mostró una estructura factorial congruente con la teoría en los análisis factoriales exploratorios y confirmatorios, siendo evidencias de validez de constructo del instrumento. Se observaron además niveles en general adecuados de consistencia interna. Los resultados descriptivos de esta medición muestran también resultados similares a los reportados por Cruz et al. (2005) hace ya 10 ańos, con promedios que se ubican casi en su totalidad por debajo de la media teórica, encontrándose en general puntajes significativamente más altos para los hombres.

Que el sexismo se asocie positivamente con el ejercicio de la violencia solo para los hombres es congruente con la concepción del sexismo ambivalente como un sistema de creencias que perpetúa el estatus quo de dominio de los hombres sobre las mujeres (Glick y Fiske, 2001). Es congruente también que el sexismo hostil y la intimidad heterosexual tengan efectos positivos sobre las dos formas de comunicación violenta evaluadas. Primero, el sexismo hostil, estaría dirigido hacia aquellas mujeres que desafían las normas socioculturales. Glick et al. (1997) señalan que algunos hombres suelen mirar a las mujeres que se dedican primordialmente a sus carreras como una competencia, y sentir hacia ellas temor, envidia o resentimiento. La agresión dirigida hacia ellas sería entonces una forma de manifestar estos sentimientos.

Respecto al sexismo benevolente, si bien presentó efectos negativos hacia la violencia, esto no debe interpretarse como un factor protector, pues tanto el sexismo hostil como el benevolente son formas de eliminar las resistencias de las mujeres, en el primer caso a través del castigo y en el segundo a través de reforzadores por cumplir con un estereotipo (Glick y Fiske, 2001).

Es necesario considerar, primero, que la intimidad heterosexual forma parte del sexismo y presenta efectos positivos hacia ambas formas de violencia, y segundo, que el sexismo benevolente guarda fuertes relaciones positivas con el sexismo hostil. Esto significaría que 
los componentes diferenciación complementaria de género y paternalismo protector podrían reducir en los hombres la tendencia de ejercer hacia sus parejas estas formas de comunicación destructiva, pero únicamente cuando estas creencias de complementariedad y protección hacia las mujeres se encuentran desvinculadas de los otros elementos del sexismo. Sin embargo, las fuertes asociaciones entre ellas permiten señalar que esto es poco probable.

Es quizá en ese contexto que paternalismo protector y diferenciación complementaria de género representen un factor que reduce el ejercicio de la comunicación destructiva de los hombres hacia sus parejas, pero ¿funcionaría igual para hombres que tienen una pareja que no aceptan la protección o los roles complementarios? Esta pregunta no puede responderse desde los datos aquí presentados, aunque los resultados de Glick et. al (2002) han mostrado que el sexismo hostil se asocia la justificación de la violencia doméstica contra las mujeres, percibiéndolas como responsables de provocarla con sus acciones, descartando además un factor de protección del sexismo benevolente.

Sobre el TESN, se plantearon dos posibles explicaciones de sus efectos como un facilitador de la violencia hacia la pareja, previamente encontrados en los hombres (Hanby et al., 2012). Los resultados apoyan la hipótesis del TESN como una característica individual que promueve la violencia verbal, mostrando asociaciones positivas con la intimidad heterosexual, soportando la hipótesis de que el TESN incrementa la dependencia hacia la pareja (Darcy et al., 2005), que asociada a la falta de recursos personales y la anuencia de la sociedad podría legitimar la violencia.

Aunque en menor medida y de forma más aislada, los efectos del TESN se observaron también en las mujeres. Dado que los reactivos de intimidad heterosexual refieren una dependencia de los hombres hacia las mujeres y al no contar con una medición de la dependencia de las mujeres hacia los hombres, no es posible saber si esta misma ruta TESN-dependencia-violencia está también presente en las mujeres, hipótesis que tendrá estudiarse posteriormente. 
Estos resultados deben interpretarse también a la luz de las limitaciones del presente estudio, identificándose como las más importantes la disparidad en el número de hombres y mujeres, y el hecho de que la muestra está compuesta de estudiantes de la Universidad más grande de México, lo cual constituye una muestra muy particular. Si bien los resultados descriptivos se asemejan a los obtenidos en estudios previos, no es posible garantizar que esta muestra representa a la población mexicana en general. Para ello será necesario obtener muestra más diversas obtenidas mediante estrategias de muestreo representativas. También debe considerarse que, dadas las edades de los participantes, las relaciones de pareja que se presentan aquí refieren a noviazgos. Aunque se han presentado ya datos sobre la gravedad de la violencia en el noviazgo, queda pendiente como una futura línea de investigación realizar un esfuerzo equivalente de investigación en parejas que cohabiten. En todo caso, los hallazgos del presente estudio sientan un precedente valioso y permiten claridad sobre los pasos a seguir en ese camino.

\section{Referencias}

Alonso, M. B., Moreno, M. y García-Baamonde, M. (2010). Revisión teórica del maltrato psicológico en la violencia conyugal. Psicologia y Salud, 20(1), 65-75.

Arbuckle, J. L. (2014). Amos (Version 23.0) [Programa de cómputo]. Chicago: IBM SPSS.

Bentler, P. M. \& Bonett, D. G. (1980). Significance tests and goodnessof-fit in the analysis of covariance structures. Psychological Bulletin, 88(3), 588-606. https://doi.org/10.1037/0033-2909.88.3.588

Coker, A.L, Davis, K. E., Arias, I., Desai, S., Sanderson, M., Brandt, H. \& Smith, P. (2002). Physical and mental health effects of intimate partner violence for men and women. American Journal of Preventive Medicine, 23(4), 260-268. https://doi.org/10.1016/ S0749-3797(02)00514-7 
Cruz, C., Diaz-Loving, R., Padilla J., Díaz, P., González, I. y Oropeza, R. (2015). Efectos del temor a la evaluación social negativa en interacción con parejas potenciales: estudio experimental del primer encuentro. Revista de Psicología Social, 30(1) 1-30. https://doi.org/10.1080/02134748.2014.987502

Cruz, T. C., Zempoaltecatl, A. V., y Correa, E. F., (2005). Perfiles de sexismo en la Ciudad de México: Validación del cuestionario de medición del Sexismo Ambivalente. Enseñanza e investigación en Psicología, 10(2), 381-395.

Darcy, K., Davila, J., \& Beck, J. G. (2005). Is social anxiety associated with both interpersonal avoidance and interpersonal dependence? Cognitive Therapy and Research, 29(2), 171-186. https:// doi.org/10.1007/s10608-005-3163-4

Expósito, F., Moya, M. C., \& Glick, P. (1998). Sexismo ambivalente: medición y correlatos. Revista de Psicología Social, 13(2), 159-169. https://doi.org/10.1174/021347498760350641

Fiske, S. T., Xu, J. y Cuddy, A. C. (1999). (Dis)Respecting versus (Dis) Liking: Status and interdependence predict ambivalent stereotypes of competence and warmth. Journal of Social Issues, 55(3), 473-489. https://doi.org/10.1111/0022-4537.00128

Follingstad, D.R., Rutledge, L.L., Berg, B.J., Hause, E.S. y Polek, D.S. (1990). The role of emotional abuse in physically abusive relationships. Journal of Family Violence, 5(2), 107-120. https://doi. org/10.1007/BF00978514

Garaigordobil, M., Aliri, J., \& Martínez-Valderrey, V. (2013). Justificación de la violencia durante la adolescencia: Diferencias en función de variables sociodemográficas. European Journal of Education and Psychology, 6(2). https://doi.org/10.30552/ejep. v6i2.96

García-Moreno, C. (2000). Violencia contra la mujer. Género y equidad en la salud. OPS/OMS. Washington: Harvard Center for Population and Development Studies.

Glick, P., Diebold, J., Bailey-Warner, B., \& Zhu, L. (1997) The two faces of Adam: Ambivalent sexism and polarized attitudes 
toward women. Personality and Social Psychology Bulletin, 23, 1323-1334. https://doi.org/10.1177/01461672972312009

Glick, P., \& Fiske, S. T. (1996). The ambivalent sexism inventory: Differentiating hostile and benevolent sexism. Journal of Personality and Social Psychology, 70(3), 491-512. https://doi. org/10.1037/0022-3514.70.3.491

Glick, P., \& Fiske, S. T. (2001). An ambivalent alliance: Hostile and benevolent sexism as complementary justifications for gender inequality. American Psychologist, 56(2), 109. https://doi. org/10.1037/0003-066X.56.2.109

Glick, P., Sakalli-Ugurlu, Ferreira, M. \& Aguiar de Souza, N. (2002). Ambivalent sexism and attitudes toward wife abuse in Turkey and Brazil. Psychology of Women Quarterly, 26, 292-297. https:// doi.org/10.1111/1471-6402.t01-1-00068

Hanby, M. S. R., Fales, J., Nangle, D. W., Serwik, A. K. \& Hedrich, U. J. (2012). Social anxiety as a predictor of dating aggression. Journal of Interpersonal Violence, 27(10), 1867-1888. https:// doi.org/10.1177/0886260511431438

Instituto Mexicano de la Juventud. (2008). Encuesta Nacional de Violencia en el Noviazgo. México: IMJ.

Instituto Nacional de Salud Pública. (2003). Encuesta Nacional sobre Violencia contra las Mujeres 2003. Cuernavaca, México: INSP.

Instituto Nacional de Salud Pública. (2006). Encuesta Nacional sobre Violencia contra las Mujeres 2006. Cuernavaca, México: INSP.

INEGI. (2013). Panorama de violencia contra las mujeres en los Estados Unidos Mexicanos. ENDIREH 2011. México: INEGI.

Kline, R. (2011). Principles and practice of structural equation modelling. New York: The Guilford Press.

Klonis, S. C., Plant, E. A., \& Devine, P. G. (2005). Internal and external motivation to respond without sexism. Personality and Social Psychology Bulletin, 31(9), 1237-1249. https://doi. org/10.1177/0146167205275304

Lambert, A. J., Payne, B. K., Jacoby, L. L., Shaffer, L. M., Chasteen, A. L., \& Khan, S. R. (2003). Stereotypes as dominant responses: 
on the "social facilitation" of prejudice in anticipated public contexts. Journal of Personality and Social Psychology, 84(2), 277. https://doi.org/10.1037/0022-3514.84.2.277

Leary, M. (1983). A Brief Version of the Fear of Negative Evaluation Scale. Personality and Social Psychology Bulletin, 9(3), 371 - 375. https://doi.org/10.1177/0146167283093007

Levy, J. \& Varela, M. (2003). Análisis multivariable para las ciencias sociales. Madrid: Person. Prentice Hall.

Overall, N. C., Sibley, C. G., \& Tan, R. (2011). The costs and benefits of sexism: resistance to influence during relationship conflict. Journal of Personality and Social Psychology, 101(2), 271. https:// doi.org/10.1037/a0022727

Plant, E. A., \& Devine, P. G. (1998). Internal and external motivation to respond without prejudice. Journal of Personality and Social Psychology, 75(3), 811. https://doi. org/10.1037/0022-3514.75.3.811

Programa de Naciones Unidad para el Desarrollo (2017). Indicadores de Desarrollo Humano y Género en México: nueva metodología. Recuperado de http://www.mx.undp.org/content/mexico/es/ home/library/poverty/indicadores-de-desarrollo-humano-ygenero-en-mexico--nueva-metod.html

Rottenbacher de Rojas, J. M. (2012). Relaciones entre el sexismo ambivalente, el conservadurismo político y la rigidez cognitiva en una muestra de habitantes de la ciudad de Lima. Psicología desde el Caribe, 29(2), 229-256.

Sánchez Aragón, R. \& Díaz Loving, R. (2003). Patrones y estilos de comunicación de la pareja: Diseño de un inventario. Anales de Psicología, 19(2), 257-277. https://doi.org/10.5354/0719-0581 .2002 .17285

Torres, M. (2001). La violencia en casa. México: Paidós.

Watson, D. \& Friend, R. (1969). Measurement of social-evaluative anxiety. Journal of Consulting and Clinical Psychology, 33(4), 448-457. https://doi.org/10.1037/h0027806 
Welch, B. L. (1947). The generalization of student's' problem when several different population variances are involved. Biometrika, 34(1/2), 28-35. https://doi.org/10.2307/2332510

WHO (2013). Global and regional estimates of violence against women: prevalence and health effects of intimate partner violence and nompartner sexual violence. Geneva: WHO.

Zimmerman, D. W. (2004). A note on preliminary tests of equality of variances. British Journalof MathematicalandStatisticalPsychology, 57(1), 173-181. https://doi.org/10.1348/000711004849222

Recibido: 22/03/2018 Revisado: 17/09/2018 Aceptado: 18/09/2018 